\title{
Joint-space Impedance Control using Intrinsic Parameters of Compliant Actuators and Inner Sliding Mode Torque Loop
}

\author{
Gianluca Garofalo, Alexander Werner, Florian Loeffl and Christian Ott
}

\begin{abstract}
A new control law for joint-space impedance in robots with variable impedance actuators is presented. The objective is achieved using reduced information on high order derivatives compared to standard approaches, therefore leading to more reliable interactions with unknown environments. Most importantly, the impedance characteristic is given by the real stiffness and damping coefficients of each actuator, therefore updating strategies of the latter directly modify the system response obtained on the link side. The method is evaluated both in simulations and experiments. Additionally, the control law is also adapted for systems with series elastic actuators.
\end{abstract}

\section{INTRODUCTION}

In pursuing an era in which robots and humans can interact, researchers have soon realized that these human friendly robots need to look very different from the typical industrial ones. Lightweight design and compliant features are required in order to reach the expected performance and safe interactions with unknown environments (especially with humans). To overcome the technological limit reached with conventional rigid robotic actuation, both Series Elastic Actuators (SEA) [1] and many Variable Impedance Actuators (VIA) have been designed in the last decades to achieve efficiency, robustness against external perturbations and adaptability during interactions [2]. While in a SEA the spring in the coupling mechanism between the motor and the link is constant, in a VIA the compliant response of the system is adjustable. Nevertheless, the challenging design phase of the control laws for such complex systems has led to closed-loop systems which are typically incapable of satisfying all these objectives. As a viscoelastic element is interposed between the motor and the actuated link, these systems are underactuated. The control strategies, therefore, require often the knowledge of the time derivatives of the link position (e.g. the link acceleration and jerk) in order to control the output of the system [3]. These values can be obtained either through filtering or model based approaches. It is clear that interactions with the environment are critical for these controllers, since there will be discontinuities in the acceleration signal and often the control gains have to be considerably lowered with a consequent decrease in the performance. This point has been recently addressed in [4]. Therein, it is shown how using a damper in parallel to a spring, as in Fig. 1, can be beneficial to control the torque produced by the actuator to a given desired value. This is the case especially in presence of impacts between the robot and

The authors are with the Institute of Robotics and Mechatronics, German Aerospace Center (DLR), Wessling, Germany. gianluca.garofalo(at)dlr.de

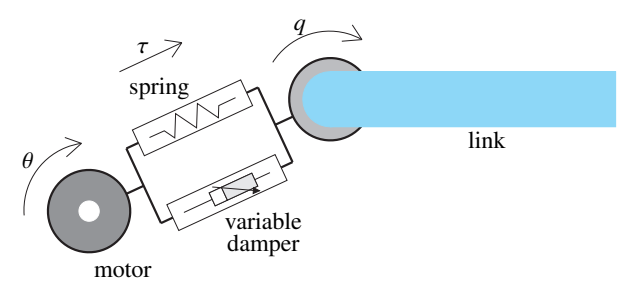

Fig. 1. Conceptual schematic of the type of VIA considered in the paper, together with the indication of torque and position variables.

the environment, i.e. in case of discontinuous acceleration. The authors compare the performance of such an actuator to those of a more classic SEA and motivate the improvement by the decreased relative degree of the torque error from $r=2$ for a SEA to $r=1$ in case of additional physical damping. Loosely speaking, the presence of the damper provides a more direct way to influence the link, rather than relying only on the spring. The results in [4] are not the first ones on this topic. An excellent work is [5], which points the reader to many related ones (e.g. [6]-[9]) and describes the steps in the design of a SEA enhanced with a variable damper. Moreover, the authors show that a VIA with variable damping can outperform a SEA, looking at force/torque exchange and energy consumption.

The work presented in [4] is the most closely related to the approach proposed in this paper and the reader is referred to [10], [11] for a review on torque control of SEAs. Nevertheless, although the control law in [4] shares the same objective as in this paper of tracking a desired trajectory for the links, it still suffers from three main shortcomings, which are addressed here. Firstly, it needs a direct feedback of the link acceleration and uses high order derivatives of the dynamic matrices. Therefore, such a control law is more sensitive to noise and less computationally efficient than one which is only feeding back the state of the robot. Secondly, due to the cascaded structure of the closed-loop system, the analysis carried on in [4] to show the asymptotic convergence of the inner torque loop is not sufficient in order to draw conclusions on the stability of the overall system; especially in case of time-varying signals. Finally, it is unclear how the physical spring and damper can be optimally used for the task that the robot has to solve and, consequently, no insights are provided on how to modify the value of the damping online nor the impact of the time-variant damping on the stability.

The control objective in this work is to guarantee that in a system composed of joints as sketched in Fig. 1, where the motors are connected to the links through a spring and 
a variable damper, the links follow a desired trajectory. It is also required that the closed-loop system exhibits a compliant behavior during physical interaction. Therefore, the controller will realize a link-side impedance. To this end, several contributions are presented in this paper, that can be summarized as follows. The link-side impedance is defined by the values of the real damper and spring of the VIA, rather than virtual ones introduced by the control action (like in conventional strategies). Standard approaches completely reshape by control action the intrinsic torque produced by the VIA [3], [4], [11]. Therefore, after convergence of the torque error, the physical stiffness and damping have no influence on the link-side behavior. In contrast, the proposed approach uses the control action only to provide the feedforward terms necessary to track a desired trajectory, while the feedback action is realized through the parameters of the VIA. This is a key aspect of this work, as it results in two important consequences. Firstly, changes of the intrinsic parameters of the VIA directly modify the closed-loop system response. This topic is still not well investigated in the robotic community and it is an important contribution of this paper. Secondly, compared to conventional approaches, the acceleration of the links appears only as a dependency in the derivative of the feedforward term, hence it is not amplified by any controller gains. Moreover, the torque error in the inner torque loop of the control action will be shown to converge in finite time to zero, by applying sliding-mode techniques proposed recently in the control community [12]. As a result, after a finite-time, the closed loop is reduced to a classic rigid-body dynamics. Finally, a variation of the controller is presented for systems in which the damping element is missing, i.e. for classic SEA.

The paper is organized in two main parts. In Section II, the design idea and theoretical contribution are presented; while in Section III the controller is evaluated in simulations and experiments. Finally, Section IV summarizes the work.

\section{A. Notation and model}

The considered robotic systems are modeled by the nonlinear differential equations (reduced elastic-joint model [13]):

$$
\begin{aligned}
& \boldsymbol{M}(\boldsymbol{q}) \ddot{\boldsymbol{q}}+\boldsymbol{C}(\boldsymbol{q}, \dot{\boldsymbol{q}}) \dot{\boldsymbol{q}}+\boldsymbol{g}(\boldsymbol{q})=\boldsymbol{\tau}+\boldsymbol{J}_{e}^{T}(\boldsymbol{q}) \boldsymbol{w}_{e} \\
& \boldsymbol{B} \ddot{\boldsymbol{\theta}}+\boldsymbol{\tau}=\boldsymbol{\tau}_{m}+\boldsymbol{\tau}_{f} \\
& \boldsymbol{\tau}=\boldsymbol{D}(t)(\dot{\boldsymbol{\theta}}-\dot{\boldsymbol{q}})+\boldsymbol{K}(\boldsymbol{\theta}-\boldsymbol{q})
\end{aligned}
$$

where $\boldsymbol{\theta}, \dot{\boldsymbol{\theta}} \in \mathbb{R}^{n}$ ( $n$ is the number of joints) are the motor positions and velocities, which constitute together with link positions and velocities $\boldsymbol{q}, \dot{\boldsymbol{q}} \in \mathbb{R}^{n}$ the whole state of the robot. It is used $\boldsymbol{M} \in \mathbb{R}^{n \times n}$ to denote the symmetric and positive definite link-side inertia matrix, $\boldsymbol{C} \in \mathbb{R}^{n \times n}$ a Coriolis matrix satisfying $\dot{\boldsymbol{M}}=\boldsymbol{C}+\boldsymbol{C}^{T}$ and $\boldsymbol{g} \in \mathbb{R}^{n}$ the gravity torque vector. On the motor side, $\boldsymbol{B} \in \mathbb{R}^{n \times n}$ is the constant diagonal inertia matrix of the motors. The torques $\tau_{m} \in \mathbb{R}^{n}$ produced by the motors are an input to the system, together with the external torques given by $\boldsymbol{\tau}_{e}=\boldsymbol{J}_{e}^{T} \boldsymbol{w}_{e}$, where the external wrenches are stacked in $\boldsymbol{w}_{e} \in \mathbb{R}^{6 m}, m$ is the number of links in contact and
$\boldsymbol{J}_{e} \in \mathbb{R}^{n \times 6 m}$ the correspondent Jacobian matrix. Finally, $\boldsymbol{\tau}_{f}$, $\tau \in \mathbb{R}^{n}$ are the perturbing torques and the torques applied to the links, respectively. The latter are the sum of the torques produced by the springs and the dampers, being $\boldsymbol{K} \in \mathbb{R}^{n \times n}$ the constant diagonal joint stiffness matrix and $\boldsymbol{D} \in \mathbb{R}^{n \times n}$ the variable diagonal joint damping matrix.

The input of the system is allowed to be discontinuous. In this case, the differential equation with locally bounded Lebesgue-measurable right-hand side is understood in the sense of Filippov [14] and the absolutely continuous solutions satisfy the differential inclusion almost everywhere.

\section{THE SPLIT TORQUE CONTROLLER}

The control goal is to track a desired trajectory for free motions, i.e. for $\boldsymbol{w}_{e}=\mathbf{0}$ then $\boldsymbol{q} \rightarrow \boldsymbol{q}_{d}$ as $t \rightarrow \infty$. Additionally, the closed-loop system compliance should dictate the deviation from the desired trajectory during physical interactions.

\section{A. Actuators with adjustable joint damping}

To derive the first controller proposed in this paper, the model (1) is firstly rewritten (omitting the dependencies) as

$$
\begin{aligned}
& \boldsymbol{M} \ddot{\boldsymbol{q}}+(\boldsymbol{C}+\boldsymbol{D}) \dot{\boldsymbol{q}}+\boldsymbol{K} \boldsymbol{q}+\boldsymbol{g}=\boldsymbol{\tau}_{\theta}+\boldsymbol{J}_{e}^{T} \boldsymbol{w}_{e}, \\
& \boldsymbol{B} \ddot{\boldsymbol{\theta}}+\boldsymbol{\tau}_{\theta}-\boldsymbol{D} \dot{\boldsymbol{q}}-\boldsymbol{K} \boldsymbol{q}=\boldsymbol{\tau}_{m}+\boldsymbol{\tau}_{f}, \\
& \boldsymbol{\tau}_{\theta}=\boldsymbol{D} \dot{\boldsymbol{\theta}}+\boldsymbol{K} \boldsymbol{\theta}
\end{aligned}
$$

in which it has been used the identity $\boldsymbol{\tau}=\boldsymbol{\tau}_{\theta}-\boldsymbol{D} \dot{\boldsymbol{q}}-\boldsymbol{K} \boldsymbol{q}$, i.e. the torque $\tau$ has been split in the terms depending only on the motors and those depending only on the links (hence the name split torque control). It is easily verified that if

$$
\boldsymbol{\tau}_{\theta}=\boldsymbol{\tau}_{d}:=\boldsymbol{g}+\boldsymbol{M} \ddot{\boldsymbol{q}}_{d}+(\boldsymbol{C}+\boldsymbol{D}) \dot{\boldsymbol{q}}_{d}+\boldsymbol{K} \boldsymbol{q}_{d},
$$

then it is realized the classic joint-space impedance [15]:

$$
\boldsymbol{M} \ddot{\tilde{\boldsymbol{q}}}+(\boldsymbol{C}+\boldsymbol{D}) \dot{\tilde{\boldsymbol{q}}}+\boldsymbol{K} \tilde{\boldsymbol{q}}=\boldsymbol{J}_{e}^{T} \boldsymbol{w}_{e},
$$

where $\tilde{\boldsymbol{q}}:=\boldsymbol{q}-\boldsymbol{q}_{d}$ denotes the position error. Compared to conventional methods, the impedance characteristic is given by the link-side inertia and the real parameters of the actuator, rather than virtual values introduced by the control action. Therefore, an adjusting law for the variable damping matrix $\boldsymbol{D}(t)$ directly reflects in a correspondent change of the link-side impedance behavior and system response. Since enforcing (4) guarantees the solution of the control objective, in the remainder of the paper the goal is to choose $\tau_{m}$ such that (3), i.e. $\tau_{\theta}=\tau_{d}$, is satisfied. To create an inner torque loop that realizes this new objective, the first step is to remove the effect of the links in $(2 b)$, by choosing

$$
\tau_{m}=-\boldsymbol{D} \dot{\boldsymbol{q}}-\boldsymbol{K} \boldsymbol{q}+\boldsymbol{u},
$$

which leads to

$$
\boldsymbol{B} \ddot{\boldsymbol{\theta}}+\boldsymbol{\tau}_{\theta}=\boldsymbol{u}+\boldsymbol{\tau}_{f},
$$

where now the new control input $\boldsymbol{u} \in \mathbb{R}^{n}$ has to be chosen in such a way that (3), and therefore (4), is satisfied. Differentiating (2c) with respect to time, it follows:

$$
\ddot{\boldsymbol{\theta}}=\boldsymbol{D}^{-1}\left(\dot{\boldsymbol{\tau}}_{\theta}-\dot{\boldsymbol{D}} \dot{\boldsymbol{\theta}}-\boldsymbol{K} \dot{\boldsymbol{\theta}}\right) \text {. }
$$


Choosing $\boldsymbol{u}=\boldsymbol{B} \boldsymbol{D}^{-1}[z-(\dot{\boldsymbol{D}}+\boldsymbol{K}) \dot{\boldsymbol{\theta}}]$ and substituting (7) into (6) leads to

$$
\dot{\boldsymbol{\tau}}_{\theta}+\boldsymbol{D} \boldsymbol{B}^{-1} \boldsymbol{\tau}_{\theta}=\boldsymbol{z}+\boldsymbol{D} \boldsymbol{B}^{-1} \boldsymbol{\tau}_{f}
$$

where once again $z \in \mathbb{R}^{n}$ is a new control input. In order to achieve a generalized super-twisting algorithm [16] for the torque error (which guarantees its finite-time convergence by means of a continuous control input), the control action for the system (8) is chosen as

$$
\begin{aligned}
& z=\dot{\boldsymbol{\tau}}_{d}+\boldsymbol{D} \boldsymbol{B}^{-1} \boldsymbol{\tau}_{d}-\boldsymbol{T}|\tilde{\tau}|^{\frac{1}{2}} \operatorname{sgn}(\tilde{\boldsymbol{\tau}})+\boldsymbol{\sigma}, \\
& \dot{\boldsymbol{\sigma}}=-\boldsymbol{S} \operatorname{sgn}(\tilde{\boldsymbol{\tau}})-\boldsymbol{P} \tilde{\boldsymbol{\tau}},
\end{aligned}
$$

where $\tilde{\boldsymbol{\tau}}:=\boldsymbol{\tau}_{\theta}-\boldsymbol{\tau}_{d}$ denotes the torque error, while the diagonal matrices $\boldsymbol{S}, \boldsymbol{T}, \boldsymbol{P} \in \mathbb{R}^{n \times n}$ are positive definite and satisfy the set of inequalities given in [16] (see also section II-C). Both $|\tilde{\tau}|^{\frac{1}{2}}$ and $\operatorname{sgn}(\tilde{\tau})$ have to be understood as element-wise applications of the correspondent operator. Finally, taking into account the expressions of the intermediate control input, the final control law is:

$$
\begin{aligned}
\boldsymbol{\tau}_{m}= & \boldsymbol{\tau}_{d}-\boldsymbol{D} \dot{\boldsymbol{q}}-\boldsymbol{K} \boldsymbol{q}+ \\
& +\boldsymbol{B} \boldsymbol{D}^{-1}\left[\dot{\boldsymbol{\tau}}_{d}-\boldsymbol{T}|\tilde{\boldsymbol{\tau}}|^{\frac{1}{2}} \operatorname{sgn}(\tilde{\boldsymbol{\tau}})+\boldsymbol{\sigma}-(\dot{\boldsymbol{D}}+\boldsymbol{K}) \dot{\boldsymbol{\theta}}\right], \\
\dot{\boldsymbol{\sigma}}= & -\boldsymbol{S} \operatorname{sgn}(\tilde{\boldsymbol{\tau}})-\boldsymbol{P} \tilde{\boldsymbol{\tau}},
\end{aligned}
$$

which leads to the closed loop system

$$
\begin{aligned}
& \boldsymbol{M} \ddot{\tilde{\boldsymbol{q}}}+(\boldsymbol{C}+\boldsymbol{D}) \dot{\tilde{\boldsymbol{q}}}+\boldsymbol{K} \tilde{\boldsymbol{q}}=\tilde{\boldsymbol{\tau}}+\boldsymbol{J}_{e}^{T} \boldsymbol{w}_{e}, \\
& \dot{\boldsymbol{\tau}}=-\boldsymbol{D} \boldsymbol{B}^{-1} \tilde{\boldsymbol{\tau}}-\boldsymbol{T}|\tilde{\tau}|^{\frac{1}{2}} \operatorname{sgn}(\tilde{\boldsymbol{\tau}})+\boldsymbol{s}, \\
& \dot{\boldsymbol{s}}=-\boldsymbol{S} \operatorname{sgn}(\tilde{\boldsymbol{\tau}})-\boldsymbol{P} \tilde{\boldsymbol{\tau}}+\boldsymbol{\rho}_{2},
\end{aligned}
$$

having defined: $\boldsymbol{s}:=\boldsymbol{\sigma}+\boldsymbol{\rho}_{3}, \rho_{2}:=\dot{\rho}_{3}$ and $\rho_{3}:=\boldsymbol{D} \boldsymbol{B}^{-1} \tau_{f}$. Considerations about the stability of the closed-loop system will be drawn in section II-C. It is important to highlight that, unlike other control laws for SEA and VIA, (10) contains no direct feedback of the link acceleration, i.e. $\ddot{q}$ does not directly appear in (10), but only as a dependency of the derivative of the Coriolis matrix in $\dot{\tau}_{d}$. This is part of the reason why the control law results highly insensitive to the availability of $\ddot{\boldsymbol{q}}$ in section III.

Since the control action contains a term which is inversely proportional to the damping, it is not applicable for SEA. In the introduction it was mentioned that SEA can be outperformed by actuators with a variable damping [4], [5]. Nevertheless, a specialization of the control law is presented in section II-B for the latter class of systems.

\section{B. Application to series elastic actuators}

In this section, the model under consideration is:

$$
\begin{aligned}
& \boldsymbol{M} \ddot{\boldsymbol{q}}+\boldsymbol{C} \dot{\boldsymbol{q}}+\boldsymbol{K} \boldsymbol{q}+\boldsymbol{g}=\boldsymbol{\tau}_{\theta}+\boldsymbol{J}_{e}^{T} \boldsymbol{w}_{e}, \\
& \boldsymbol{B} \ddot{\boldsymbol{\theta}}+\boldsymbol{\tau}=\boldsymbol{\tau}_{m}+\boldsymbol{\tau}_{f}, \\
& \boldsymbol{\tau}=\boldsymbol{K}(\boldsymbol{\theta}-\boldsymbol{q})
\end{aligned}
$$

in which, with a slight abuse of notation, $\boldsymbol{\tau}_{\theta}=\boldsymbol{K} \boldsymbol{\theta}$. Having the model in this form, one can easily verify that if

$$
\boldsymbol{\tau}_{\theta}=\tau_{d}:=\boldsymbol{g}+\boldsymbol{M} \ddot{\boldsymbol{q}}_{d}+\boldsymbol{C} \dot{\boldsymbol{q}}_{d}-\boldsymbol{D} \dot{\tilde{\boldsymbol{q}}}+\boldsymbol{K} \boldsymbol{q}_{d},
$$

then the joint impedance (4) is realized. Unlike in the previous section, this time $\boldsymbol{\tau}_{m}=\boldsymbol{\tau}+\boldsymbol{B} \boldsymbol{K}^{-1} \boldsymbol{u}$, so that (12b) reduces to a double integrator. At this point, a higher order super-twisting algorithm scheme can be adopted. To achieve the continuous singular terminal sliding mode algorithm [12] for the torque error, it is made the choice

$$
\begin{aligned}
& \boldsymbol{\phi}=\dot{\tilde{\tau}}+\boldsymbol{T}_{2}|\tilde{\tau}|^{\frac{2}{3}} \operatorname{sgn}(\tilde{\tau}), \\
& \boldsymbol{u}=\ddot{\boldsymbol{\tau}}_{d}-\boldsymbol{T}_{1}|\phi|^{\frac{1}{2}} \operatorname{sgn}(\phi)+\boldsymbol{\sigma}, \\
& \dot{\boldsymbol{\sigma}}=-\boldsymbol{T}_{3} \operatorname{sgn}(\phi),
\end{aligned}
$$

where the diagonal matrices $\boldsymbol{T}_{1}, \boldsymbol{T}_{2}, \boldsymbol{T}_{3} \in \mathbb{R}^{n \times n}$ are positive definite and satisfy the set of inequalities given in [12]. Finally, taking into account the expressions of the intermediate control variable, the final control input is:

$$
\boldsymbol{\tau}_{m}=\boldsymbol{\tau}+\boldsymbol{B} \boldsymbol{K}^{-1}\left(\ddot{\boldsymbol{\tau}}_{d}-\boldsymbol{T}_{1}|\boldsymbol{\phi}|^{\frac{1}{2}} \operatorname{sgn}(\boldsymbol{\phi})+\boldsymbol{\sigma}\right)
$$

which leads to the closed loop system

$$
\begin{aligned}
& \boldsymbol{M} \ddot{\tilde{\boldsymbol{q}}}+(\boldsymbol{C}+\boldsymbol{D}) \dot{\tilde{\boldsymbol{q}}}+\boldsymbol{K} \tilde{\boldsymbol{q}}=\tilde{\boldsymbol{\tau}}+\boldsymbol{J}_{e}^{T} \boldsymbol{w}_{e}, \\
& \ddot{\tilde{\tau}}=-\boldsymbol{T}_{1}|\boldsymbol{\phi}|^{\frac{1}{2}} \operatorname{sgn}(\boldsymbol{\phi})+\boldsymbol{s}, \\
& \dot{\boldsymbol{s}}=-\boldsymbol{S} \operatorname{sgn}(\boldsymbol{\phi})+\boldsymbol{\rho}_{2},
\end{aligned}
$$

having defined: $\boldsymbol{s}:=\boldsymbol{\sigma}+\boldsymbol{\rho}_{3}, \boldsymbol{\rho}_{2}:=\dot{\boldsymbol{\rho}}_{3}$ and $\boldsymbol{\rho}_{3}=\boldsymbol{K} \boldsymbol{B}^{-1} \boldsymbol{\tau}_{f}$ As expected, an additional derivative of the desired torque is used in this case compared to (10). Moreover, the control law in this section, unlike (10), contains a direct feedback of link acceleration and jerk due to the term $\boldsymbol{D} \dot{\tilde{\boldsymbol{q}}}$ in $\boldsymbol{\tau}_{d}$.

\section{Stability analysis}

The stability properties of the closed-loop systems (11) and (16) in case of free motion $\left(\boldsymbol{w}_{e}=\mathbf{0}\right)$, are easily derived thanks to the finite-time convergence of the torque error.

Proposition 1: Let $\boldsymbol{q}_{d}(t)$ be a smooth desired trajectory. If $\boldsymbol{\tau}_{f}$ has a known global Lipschitz constant, such that $\left|\boldsymbol{\rho}_{2}\right|<\delta_{2}$ for some constant $\delta_{2} \geq 0$, then for $\boldsymbol{w}_{e}=\mathbf{0}$ the system (11) is uniformly globally asymptotically stable, provided that the mean value $\overline{\boldsymbol{D}}$ of $\boldsymbol{D}(t)$ and the positive definite diagonal gain matrices are sufficiently large.

Proof: Rewriting $\boldsymbol{D}(t)$ as $\boldsymbol{D}(t)=\overline{\boldsymbol{D}}+\Delta \boldsymbol{D}(t)$ and defining $\rho_{1}:=\Delta \boldsymbol{D}(t) \boldsymbol{B}^{-1} \tilde{\boldsymbol{\tau}},(11 \mathrm{~b})$ and (11c) can be written as

$$
\begin{aligned}
& \dot{\tilde{\tau}}=-\bar{D} B^{-1} \tilde{\tau}-\boldsymbol{T}|\tilde{\tau}|^{\frac{1}{2}} \operatorname{sgn}(\tilde{\tau})+\boldsymbol{s}+\rho_{1}(t, \tilde{\tau}), \\
& \dot{\boldsymbol{s}}=-\boldsymbol{S} \operatorname{sgn}(\tilde{\boldsymbol{\tau}})-\boldsymbol{P} \tilde{\boldsymbol{\tau}}+\boldsymbol{\rho}_{2}(t),
\end{aligned}
$$

where $\left|\boldsymbol{\rho}_{1}\right| \leq \delta_{1}|\tilde{\boldsymbol{\tau}}|$ for some constant $\delta_{1} \geq 0$. This has the exact same form as the modified second order sliding mode (SOSML) in [16], for which robust, global finitetime stability of $(\tilde{\boldsymbol{\tau}}, \boldsymbol{\sigma})=(\mathbf{0}, \mathbf{0})$ is guaranteed if $S_{i}, P_{i}$, $T_{i}$ and $\bar{D}_{i} B_{i}^{-1}$ satisfy the inequalities given in [16], where the subscript denotes the $i$ - th entry on the diagonal. In particular, two sets of inequalities are provided for the gains of the SOSML, which can always be solved for every $\delta_{1}>0$, $\delta_{2}>0$. For the specific case in (17), using $F_{i}:=\bar{D}_{i} B_{i}^{-1}$, the 
two sets of inequalities reduce to

$$
\begin{aligned}
T_{i} & >2 \sqrt{\delta_{2}} \\
\bar{D}_{i} & >2 \max \left(\Delta D_{i}\right) \\
P_{i} & >\max \left(\frac{9}{4} \frac{T_{i}^{2} F_{i}^{2}}{S_{i}-\delta_{2}}+2 F_{i}^{2}+\frac{3}{2} F_{i} \delta_{1}, F_{i} \frac{F_{i}^{2}+3 F_{i} \delta_{1}+\frac{1}{2} \delta_{1}^{2}}{F_{i}-2 \delta_{1}}\right) \\
S_{i} & >\max \left(\delta_{2}, \frac{\left(\frac{1}{2} T_{i} \delta_{1}\right)^{2}+2 F_{i}^{2} \delta_{2}-4 T_{i}^{2} F_{i}^{2}+T_{i}^{2} F_{i} \delta_{1}}{2 F_{i}\left(F_{i}-2 \delta_{1}\right)}\right) .
\end{aligned}
$$

The stability properties of the whole system follow from its simple cascaded structure and the finite-time convergence of the always bounded torque error. For $t>T$ (i.e. when $\tilde{\boldsymbol{\tau}}=\mathbf{0}$ ), it is well known that (4) is exponentially stable in case of free motion [15], since the constant $\boldsymbol{K}$ and the timevariant $\boldsymbol{D}(t)$ are positive definite. Finally, for $t>T$ it holds:

$$
\boldsymbol{\tau}_{\theta}=\boldsymbol{\tau}_{d} \Longrightarrow \dot{\boldsymbol{\theta}}=-\boldsymbol{D}^{-1} \boldsymbol{K}\left(\boldsymbol{\theta}-\boldsymbol{K}^{-1} \boldsymbol{\tau}_{d}\right)
$$

meaning that $\boldsymbol{\theta}$ is a filtered version ${ }^{1}$ of $\boldsymbol{K}^{-1} \boldsymbol{\tau}_{d}$. This also shows that there is no unstable zero dynamics, recalling the boundedness of the dynamic matrices [17] and of $\tau_{d}$.

Similar considerations are valid for the system (16). Nevertheless, the conditions that the gains have to satisfy are much more complex [12].

\section{Practical considerations}

By removing the feedforward term $\dot{\boldsymbol{\tau}}_{d}$ in (10), $\dot{\boldsymbol{\tau}}_{d}$ can be seen as an additive term to the perturbation $\rho_{2}$. Unfortunately, this makes $\rho_{2}$ state-dependent and therefore it cannot be a priori guaranteed to have a global Lipschitz constant. In the current state, the control law already has the advantage of using no direct feedback of the link acceleration and it proved to perform well even when setting $\dot{\boldsymbol{\tau}}_{d}=\mathbf{0}$ both in simulations and experiment (see Section III).

An adjustable spring in the VIA, can be useful when the robot is required to perform different tasks, but unlike the damper that can be adjusted within the task, adaptations of the springs have to be done between tasks. This is due to the design choice of solving the joint tracking problem by enforcing the link-side behavior (4), which allows to have a time-varying damping, but requires a constant stiffness [15].

\section{VALIDATION}

The same system setup, gain values and desired trajectory for the link were considered both in the experiments and in the simulations. The latter were used to compare the proposed control laws to different existing approaches. Since the control laws in the literature typically aim at driving the torque $\tau$ in (1) to a desired value (rather than $\tau_{\theta}$ ), in this section, the torque error will be redefined as $\tau-\tau_{d}^{*}$, where

$$
\tau_{d}^{*}:=\boldsymbol{g}+\boldsymbol{M} \ddot{\boldsymbol{q}}_{d}+C \dot{\boldsymbol{q}}_{d}-\boldsymbol{D} \dot{\tilde{\boldsymbol{q}}}-\boldsymbol{K} \tilde{\boldsymbol{q}}
$$

for both the VIA and SEA case and it has the same value as the previously defined $\tilde{\tau}$.

\footnotetext{
${ }^{1}$ The filter response is modified by a change in $\boldsymbol{D}(t)$.
}

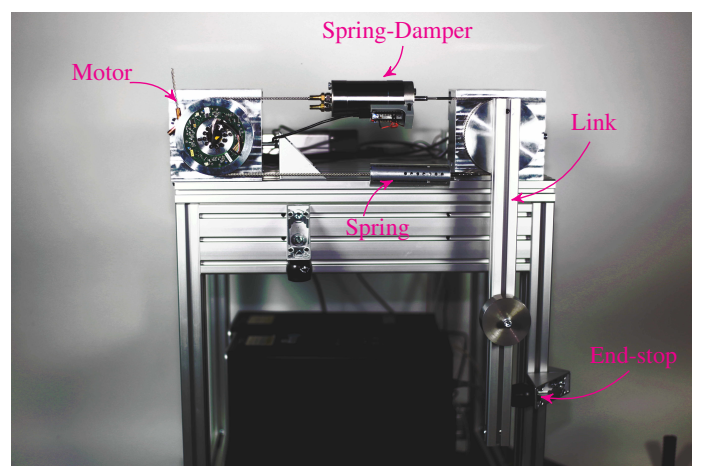

Fig. 2. Experimental setup. The link is at $q=0$, which is used as initial position. The end-stop to the right is located near to $q=0$ and it is used for the impact experiment.

TABLE I

SySTEM PARAMETERS

\begin{tabular}{ccccc} 
Motor inertia & Link inertia & Stiffness & Damping & Max torque \\
\hline$B$ & $M$ & $K$ & $D$ & $\left|\tau_{m}\right|$ \\
$1.53 \mathrm{~kg} \cdot \mathrm{m}^{2} / \mathrm{s}^{2}$ & $2.60 \mathrm{~kg} \cdot \mathrm{m}^{2} / \mathrm{s}^{2}$ & $416.16 \mathrm{~N} \cdot \mathrm{m} / \mathrm{rad}$ & $37.29 \mathrm{~N} \cdot \mathrm{m} \cdot \mathrm{s} / \mathrm{rad}$ & $100 \mathrm{~N} \cdot \mathrm{m}$ \\
\hline
\end{tabular}

\section{A. Experiments}

The experiments were conducted on a modular test setup for VIA similar to the one used in [4] and it is illustrated in Fig. 2. Three major components can be recognized: the motor, the link and the coupling mechanism between the two. The link is simply a rigid body equipped with a position sensor. As motor, a DLR LWR actuator module was used. It consists of a brushless DC motor, a harmonic drive gear (1:100), a position sensor and a torque sensor. The velocity is obtained differentiating the position signal. The maximum absolute value of the torque that can be generated by the module is $100 \mathrm{Nm}$. A current control loop allows the motor to produced the required value of the torque $\tau_{m}$. Finally, the coupling mechanism, modelled as a parallel spring-damper combination, is implemented by two counteracting elements. The first is a conventional steel spring, while the second is a recently designed spring-damper element, shown in Fig. 3. In there, two air chambers which together realize a nearly linear spring can be recognized, as well as two additional oilfilled chambers, which realize instead the viscous damper. The damping coefficient is adjustable via a servo controlled valve. The values of the parameter of the test setup are summarized in Table I. Additionally, two significant parasitic effects are present. One is the friction torque generated by the harmonic drive gear, which was estimated to be in the range of $6 \mathrm{Nm}$. The other is the friction generated by the seals of the spring-damper element, which is also roughly in that range. Finally, the torque controller was executed at $3 \mathrm{kHz}$ on a COTS computer with Linux Prempt-RT, which connects to the sensors and the drive via Ethercat ${ }^{\circledR}$.

The experiments consisted in tracking the desired link trajectory shown in orange at the bottom of Fig. 4. The link, initially close to the end-stop at nearly $q=0$, is asked to move at $-0.2 \mathrm{rad}$. After the initial step, the desired trajectory is sinusoidal and it is followed by a second step. 


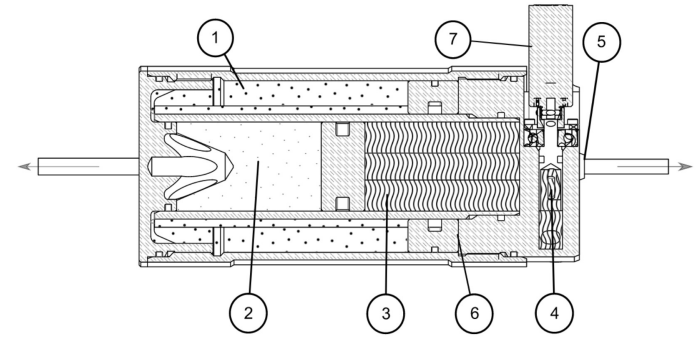

Fig. 3. The spring-damper element. The elasticity is realized by the active air chamber (1) and the linearizing air chamber (2). The viscous effect is due to the motion of the piston rod (5) pushing oil through the valve (4) between the chambers (3) and (6). Finally, the servo (7) allows to adjust the damping coefficient by acting on the variable throttle valve (4).

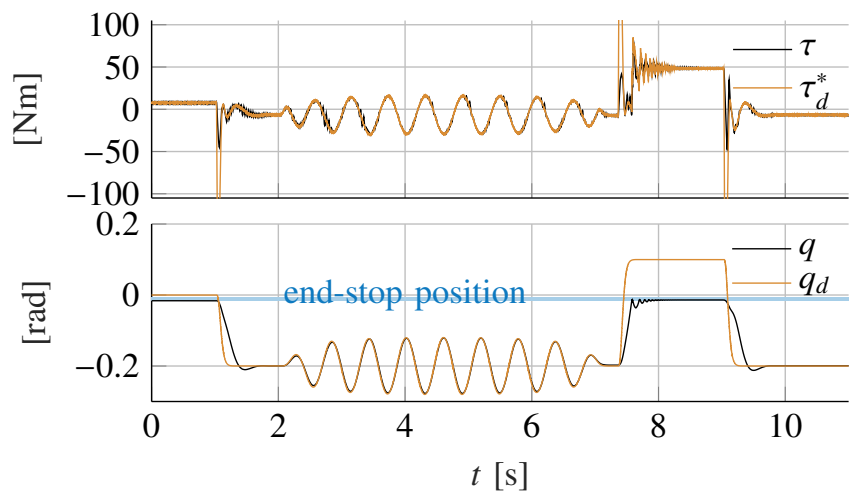

Fig. 4. Resulting joint position and torque tracking in the experiment. The deviation of $q$ from the desired value $q_{d}$ between around $7.4 \mathrm{~s}$ and $9 \mathrm{~s}$ is due to the interaction forces with the end-stop.

This time, being the desired position at $0.1 \mathrm{rad}$, i.e. beyond the end-stop, an impact will occur. Finally, the last step in the desired trajectory brings the link back to $q=-0.2 \mathrm{rad}$. The original desired trajectory was filtered to obtain a sufficiently smooth signal together with its time derivatives. The tracking performances of the controller for both the torque and link position are shown in Fig. 4. The peaks in the desired torque (corresponding to the discontinuities in the original desired position signal) have been left out from the figure to have a better scaling of the signals. Fig. 5 shows a magnified view of the link and motor velocities at the impact with the endstop. The VIA joint reduces the effects of the impact on the motor, noticeable by the smoother and reduced oscillations.

In the second experiment, unlike in the previous one and

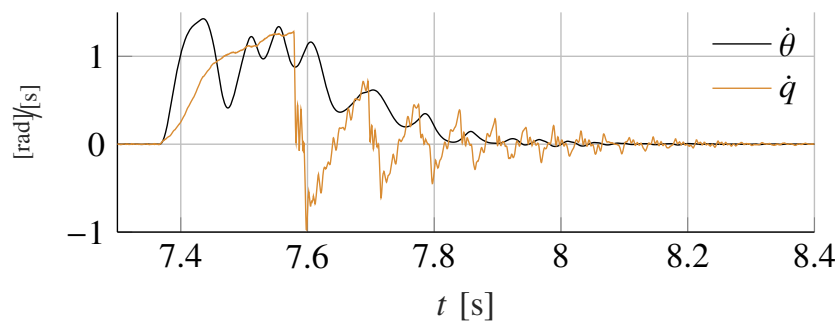

Fig. 5. Magnified view of the motor and link velocities at the impact with the end-stop. The effects of the impact on the motor are effectively reduced.

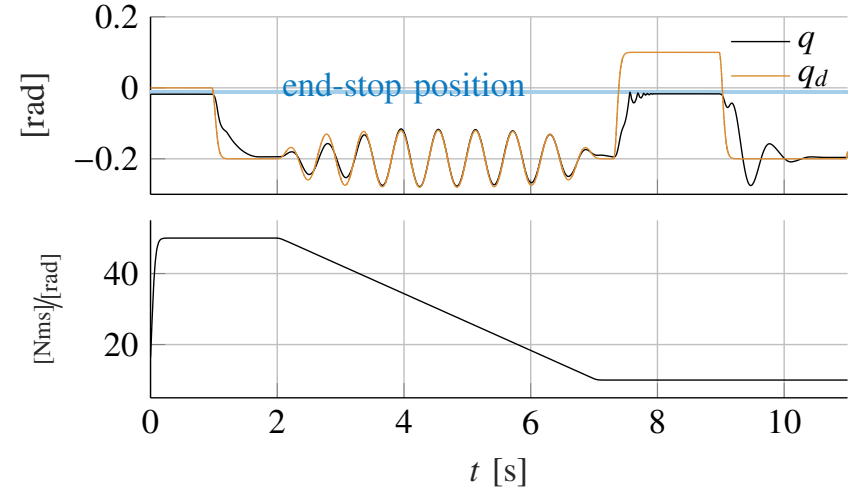

Fig. 6. Different settling time of the system with a varying $D$, unlike the behaviour obtained with a constant value in all the other tests.

all the simulations in Section III-B, the value of $D$ is not kept constant as in Table I, but changes as in Fig. 6. A substantial difference in the settling time to the steps in $q_{d}$ is obtained, as the system goes from being overdamped to underdamped. Also, the variation of $D$ does not affect the response of the system once it is already perfectly tracking the reference.

\section{B. Simulation}

The performances of the closed-loop systems (11) and (16) are compared to the control law in [4] and the cascaded torque control in [11], respectively. The gains in the first case were taken from [4], with $S, T$ and $P$ chosen such that a similar control input is obtained, shown in the top plot of Fig. 7. The tracking performances are similar, but a faster convergence is obtained with the split torque controller both for the steps and the sinusoidal part in the desired trajectory. Similar considerations hold for the SEA version, although in this case the improvement is observable only for the sinusoidal part in Fig. 8. Note that the end-stop, being not perfectly rigid, is slightly compressed by the end-effector.

The sensitivity of the split torque control and the one in [4] to the availability of the high order derivatives (i.e. $\ddot{q}$ and $\left.\dot{\tau}_{d}\right)$ is also tested by setting these values to zero and keeping all the gains unchanged. In real world scenarios, these terms could be noisy or wrongly estimated, therefore it is an important feature of the controller to show insensitivity to their values. The value of $\dot{\tau}_{d}$ was indeed set to zero in the previously described experiments. As Fig. 9 clearly shows, the tracking obtained with the control law proposed in this paper (top plot) shows no noticeable difference, while the tracking capabilities are highly compromised with the one in [4]. Although both approaches theoretically request the same signals, the split torque control law is, in this case, far less sensitive than the other to their precise availability.

\section{Conclusion}

The paper walks the reader through the derivation of a novel control law for VIA with adjustable damping. The main goal is the realization of a joint-space impedance which is defined by the intrinsic springs and dampers in the actuators. The motivation is to efficiently exploit the presence 


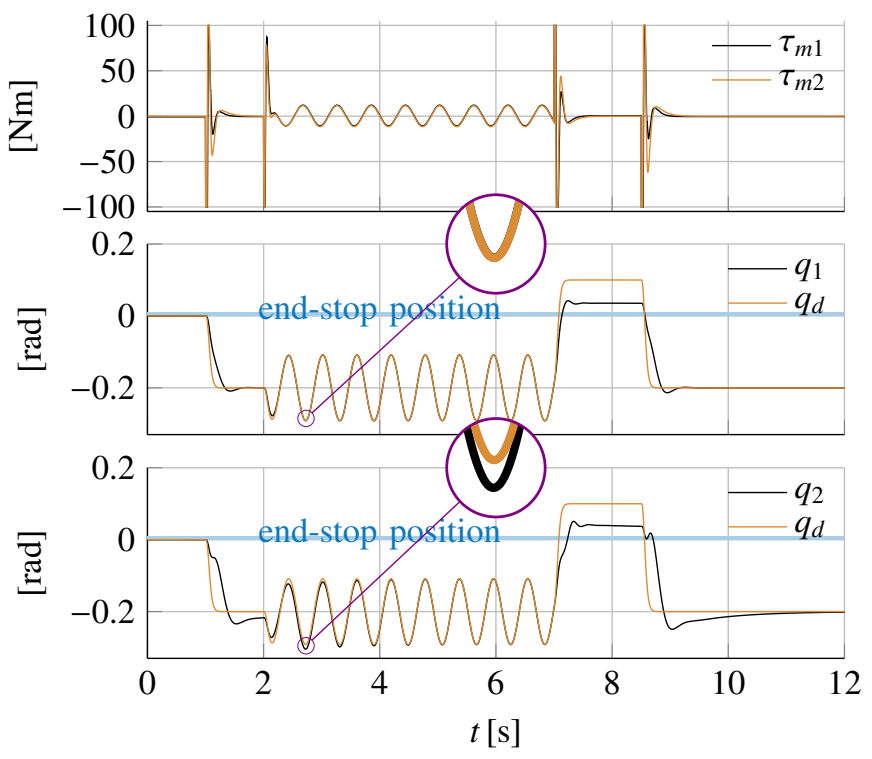

Fig. 7. Compared simulations of the split torque controller and the one in [4], labeled as 1 and 2 respectively. Although the gains were tuned to get a similar control output (top plot), a faster and better convergence is obtained with the split torque controller.

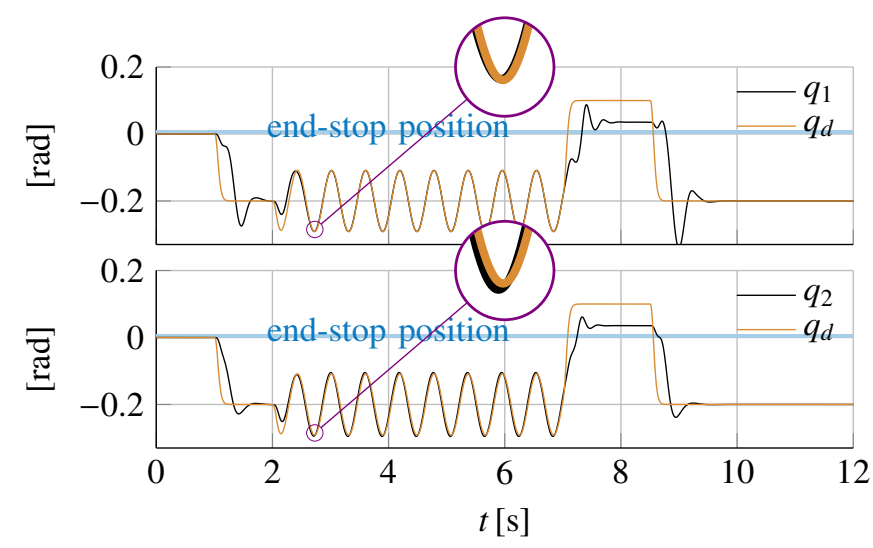

Fig. 8. Compared simulations of the system (16) and the cascaded structure in [11], labeled as 1 and 2 respectively. While the split torque controller achieves perfect tracking, this is not the case with the controller in [11].

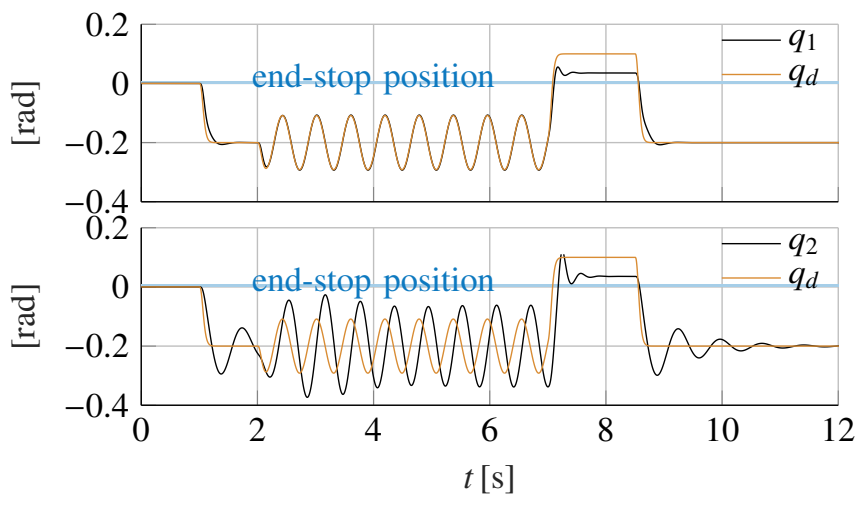

Fig. 9. Compared simulations of the split torque controller and the one in [4], labeled as 1 and 2 respectively. The values of $\ddot{q}$ and $\dot{\boldsymbol{\tau}}_{d}$ were set to zero to test the sensitivity of the controllers to the availability of these signals. The split torque controller clearly outperforms the other in this case. of such actuators in the robotic system and possibly minimize the control effort. Relying on a modified version of the supertwisting algorithm for the inner torque control loop, the controller is less sensitive to information on the higher order derivatives of the state of the robot as compared to other control laws found in the literature. Such a feature renders the controller well suited for robotic systems interacting with an unknown environment (e.g. for human-robot interaction). Lastly and most importantly, the appearance of the physical parameters in the realization of the joint impedance allows for a very intuitive and easy strategy for their adaptation; a topic still not well investigated in the robotic community.

\section{REFERENCES}

[1] G. A. Pratt and M. M. Williamson, "Series elastic actuators," in IEEE/RSJ Int. Conf. on Intelligent Robots and Systems (IROS), Pittsburgh, USA, Aug. 1995, pp. 399-406.

[2] B. Vanderborght, A. Albu-Schäffer, A. Bicchi, E. Burdet, D. G. Caldwell, R. Carloni, et al., "Variable impedance actuators: A review," Robotics and Autonomous Systems, vol. 61, no. 12, pp. 1601-1614, 2013.

[3] B. Brogliato, R. Ortega, and R. Lozano, "Globally stable nonlinear controllers for flexible joint manipulators: a comparative study," $\mathrm{Au}$ tomatica, vol. 31, no. 7, pp. 941-956, 1995.

[4] M. J. Kim, A. Werner, F. C. Loeffl, and C. Ott, "Enhancing joint torque control of series elastic actuators with physical damping," in IEEE Int. Conf. on Robotics and Automation (ICRA), Singapore, Singapore, May 2017, pp. 1227-1234.

[5] M. Laffranchi, L. Chen, N. Kashiri, J. Lee, N. G. Tsagarakis, and D. G. Caldwell, "Development and control of a series elastic actuator equipped with a semi active friction damper for human friendly robots," Robotics and Autonomous Systems, vol. 62, no. 12, pp. 18271836, 2014.

[6] C.-M. Chew, G.-S. Hong, and W. Zhou, "Series damper actuator: a novel force/torque control actuator," in IEEE/RAS Int. Conf. on Humanoid Robots, Santa Monica, USA, Nov. 2004, pp. 533-546.

[7] E. Garcia, J. C. Arevalo, G. Muñoz, and P. G. de Santos, "Combining series elastic actuation and magneto-rheological damping for the control of agile locomotion," Robotics and Autonomous Systems, vol. 59, no. 10, pp. 827-839, 2011.

[8] A. Enoch, A. Sutas, S. Nakaoka, and S. Vijayakumar, "BLUE: A bipedal robot with variable stiffness and damping," in IEEE/RAS Int. Conf. on Humanoid Robots, Osaka, Japan, Nov. 2012, pp. 487-494.

[9] A. Radulescu, M. Howard, D. J. Braun, and S. Vijayakumar, "Exploiting variable physical damping in rapid movement tasks," in 2012 IEEE/ASME International Conference on Advanced Intelligent Mechatronics (AIM), Kachsiung, Taiwan, July 2012, pp. 141-148.

[10] Y. Zhao, N. Paine, S. J. Jorgensen, and L. Sentis, "Impedance control and performance measure of series elastic actuators," IEEE Transactions on Industrial Electronics, vol. 65, no. 3, pp. 2817-2827, 2018.

[11] C. Ott, Cartesian Impedance Control of Redundant and Flexible-Joint Robots, ser. Springer Tracts in Advanced Robotics. Berlin: SpringerVerlag, 2008.

[12] L. Fridman, J. A. Moreno, B. Bandyopadhyay, S. Kamal, and A. Chalanga, Continuous Nested Algorithms: The Fifth Generation of Sliding Mode Controllers. Cham: Springer International Publishing, 2015, pp. 5-35.

[13] M. W. Spong, "Modeling and control of elastic joint robots," the ASME Journal of Dynamic Systems, Measurement, and Control, vol. 109, pp. 310-318, 1987.

[14] A. F. Filippov, Differential equations with discontinuous righthand sides, ser. Mathematics and its applications. Dordrecht: Kluwer Academic, 1988.

[15] R. M. Murray, Z. Li, and S. S. Sastry, A Mathematical Introduction to Robotic Manipulation. CRC Press, 1994.

[16] J. A. Moreno and M. Osorio, "A Lyapunov approach to second-order sliding mode controllers and observers," in IEEE Conf. on Decision and Control, Cancun, Mexico, Dec. 2008, pp. 2856-2861.

[17] C. Canudas-de-Wit, B. Siciliano, and G. Bastin, Theory of robot control. London: Springer-Verlag, 1996. 